\title{
Preparation of complex of polyaniline and acrylic ester grafting epoxy for anticorrosion and intrinsically antistatic coatings
}

\author{
Hongsheng Wang, Aiping Zhu*, Zhengxi Zhu \\ College of Chemistry and Chemical Engineering, Yangzhou University, Yangzhou, \\ 225002, P. R. China \\ *Corresponding Author: Aiping zhu
}

\begin{abstract}
In this paper, acrylic ester grafting epoxy with carboxyl groups (A-g-EP) was firstly prepared using solution polymerization. A complex of polyaniline (PANI) and A-gEP (PANI-A-g-EP) was then prepared by in-situ chemical oxidation polymerization of aniline, which pre-reacted with the carboxyl groups of A- $g$-EP. FT-IR spectra confirmed the formation of the PANI-A-g-EP complex, and it has a unique self-crosslinking structure, which comes from ester formation between epoxy and carboxyl groups on the A-gEP chain. Effects of PANI content on the glass transition temperature $\left(T_{\mathrm{g}}\right)$ and the thermal decomposition temperature $\left(T_{\mathrm{d}}\right)$ of A-g-EP were investigated. The partially oxidized emeraldine salt state of PANI in the complex was revealed by UV-Vis, FT-IR and cyclic voltammetry (CV). The antistatic property was investigated with a surface resistance measurement. Anticorrosion performances of PANI-A-g-EP complex coating on mild steel in $0.1 \mathrm{M}$ of $\mathrm{H}_{2} \mathrm{SO}_{4}$ solutions were investigated by electrochemical impedance spectroscopy (EIS). The anticorrosion and antistatic mechanisms were discussed.
\end{abstract}

Keywords: Acrylic ester grafting epoxy, Polyaniline, Antistatic property, Anticorrosion.

\section{Introduction}

Acrylics have been widely used for coatings, textiles, polishes, and other applications, since the resins typically have a remarkable hydrolytic, light, and oxidative stability [1], while epoxy resins show excellent heat resistance, adhesion, and anticorrosion [2,3]. A copolymer of acrylics and epoxies could possess advantages of both resins [4], and was regarded as a better coating material to protect metallic substrates against corrosion [8-10]. It can be synthesized by graft polymerization of acrylics to the aliphatic backbone of epoxies [6-7].

In recent years, intrinsically conducting polymers (ICPs) have gained unprecedented attractions due to their metal-like conductivity in a doped state [19, 20]. Polyaniline (PANI) is one of the most investigated ICPs because it has a low cost, easiness of synthesis, and a good physicochemical stability [17]. It has been widely applied in biosensors, storage batteries, electrochromic devices, and optical components [18]. Addition of an ICP into a coating formulation is a new strategy for anticorrosion, reducing emissions of carcinogenic heavy metal ions from metallic additives [11-14], and overcoming the difficulty of electroplating ICP films in a large area [15-16]. PANI was usually fed in a form of particles due to its infusibility and insolubility. When a PANI concentration in a coating exceeded a certain value, PANI aggregated inevitably, which can rather decrease a corrosion resistance and conductivity of the coating [21]. Armelin and co-workers [22], for instance, showed that as the PANI percentage added to an epoxy paint formulation increased from 0.3-1.5 $\mathrm{wt} \%$, the surface defect of the coating appeared. This can significantly affect the coating properties, e.g., gloss, and stain resistance. Meanwhile, such a low feed ratio along with the significant particle aggregation made PANI hard to be able to form a conductive network in the coating matrix. Thus the conductivity of the coating cannot be sufficiently improved to meet the requirement of anti-electrostatic coatings.

Considering that the conductivity of PANI can be easily affected by the stability of its half-oxidized state, it is of great importance to investigate how to obtain the stable state of PANI in its complex. This paper was 
to develop an anticorrosion and intrinsically antistatic coating material, i.e., a complex of polyaniline and acrylic ester grafting epoxy (PANI-A-g-EP). The chemical structure and redox state of PANI in the complex were studied by using FT-IR, UV-Vis and CV. A mechanism was proposed in this study to better explain the excellent anticorrosive and antistatic properties of the complex coating.

\section{Materials and methods}

\subsection{Synthesis}

2.1.1 Preparation of acrylic resin grafting epoxy (A-g-EP)

A four-necked round bottom flask was added with 6.8 $g$ of E-51 and $70 \mathrm{~g}$ of toluene with a stir at $200 \mathrm{r} \cdot \mathrm{min}^{-1}$ for ten minutes to form a solution. The temperature of above system was raised to $94{ }^{\circ} \mathrm{C}$. Then the solution of $3.40 \mathrm{~g}$ of AA, $0.34 g$ of AM, $34.48 g$ of MMA, $22.98 g$ of BA, $1.8 g$ of BPO, and $12 \mathrm{~g}$ of butanone were added dropwise to the reactor. After feeding for $3 h$, the temperature of the reaction system was increased to $97{ }^{\circ} \mathrm{C}$, and maintained for another $2 h$. Finally, the polymerization system was cooled down to $40{ }^{\circ} \mathrm{C}$. A solution of A- $g$-EP was obtained.

\subsubsection{Preparation of PANI-A-g-EP}

A-g-EP solution $(50 \mathrm{~g})$ and aniline $(0.6 \mathrm{~g}, 1.2 \mathrm{~g}$ and $2.4 \mathrm{~g}$ ) were placed into a three-necked round bottom flask with a stir at $200 \mathrm{r} \cdot \mathrm{min}^{-1}$. Then, a certain amount of BPO (the molar ratio of aniline to BPO was 1) was dissolved in $20 \mathrm{~g}$ of butanone, and was added dropwise into the reaction system. The solution was submerged in an ice-bath and reacted for another $24 \mathrm{~h}$ to obtain the PANI(3\%)-A-g-EP (which represents PANI-A-g-EP with $3 \%$ of PANI solid content), PANI(6\%)-A-g-EP and PANI(12\%)-A-g-EP complex respectively.

\section{Results and Discussion}

\subsection{Synthesis and Characterization}

The structure representation of A-g-EP is shown in Scheme 1a. A-g-EP can be synthesized by graft polymerization of the acrylic branches to the aliphatic backbone of an epoxy resin [3-5]. Free radicals were able to be generated on the hydrogen atoms on the aliphatic backbone of an epoxy by heat to initiate the graft polymerization. As a result, the epoxy and carboxyl groups can be introduced into the A-g-EP. Due to the reaction between epoxy and carboxyl groups on Scheme 1a, a selfcrosslinking A-g-EP can be obtained as shown in Scheme $1 b$.

Scheme 1c shows the synthesis of PANI-A-g-EP complex. Firstly, aniline can react with the carboxyl groups on A-g-EP through acid-base neutralization. These aniline monomers bonded to A- $g$-EP were then initiated by BPO and polymerized. After this in-situ oxidation polymerization of PANI, PANI-A-g-EP was obtained.

\subsubsection{FT-IR}

Fig. 1 shows the FT-IR spectra of A- $g$-EP and PANIA- $g$-EP. On the spectrum of A-g-EP, the peaks at 2924 and $2864 \mathrm{~cm}^{-1}$ are attributed to the stretching vibration of $-\mathrm{CH}_{3}$ and $-\mathrm{CH}_{2-} ; 1733 \mathrm{~cm}^{-1}$ belongs to the stretching vibration of $\mathrm{C}=\mathrm{O} ; 1237$ and $1147 \mathrm{~cm}^{-1}$ are symmetric stretching vibration of $\mathrm{C}-\mathrm{O}-\mathrm{C} ; 825 \mathrm{~cm}^{-1}$ is attributed to the scissoring vibration of - $\mathrm{CH}$ - on the epoxy groups; It should be noticed that there is no evident peak at $914 \mathrm{~cm}^{-1}$, which is characteristic vibration of epoxy groups, in the spectrum of A- $g$-EP. The absence of this peak indicates that the epoxy groups on A-g-EP have been reacted with carboxyl groups to form a ester crosslinked structure (Scheme 1b). FT-IR result clearly indicates the successful preparation of A- $g$-EP and there is a self-crosslinking structure in A- $g$-EP. On the spectrum of PANI-A-g-EP, the peaks at 836, 1599, 1143, 1500 , and $1310 \mathrm{~cm}^{-1}$ correspond to the $\mathrm{C}-\mathrm{H}$ bending vibration of the para substituted benzene, absorbing vibration of quinone imine $(\mathrm{N}=\mathrm{Q}=\mathrm{N})$, mode vibration of quinone imine $(\mathrm{N}=\mathrm{Q}=\mathrm{N})$, absorbing vibration of benzenoid structure(N-B-N), and stretching vibration of arylamine (Ar-N), respectively. The peak at $1733 \mathrm{~cm}^{-1}$ is attributed to stretching vibration of $\mathrm{C}=\mathrm{O}$. Compared to the spectrum of A- $g$-EP, the characteristic peaks of PANI-A-g-EP at 1599 $\mathrm{cm}^{-1}(\mathrm{~N}=\mathrm{Q}=\mathrm{N})$ and $1500 \mathrm{~cm}^{-1}(\mathrm{~N}-\mathrm{B}-\mathrm{N})$ appear, and the peaks intensity enhanced with the increase of the PANI content. These results also confirm that the formation of PANI-A-g-EP. FT-IR results further demonstrate that the PANI exists part in the oxidized pernigraniline state and the other in the emeraldine state (EB) in PANI-A-g-EP complex [23].

\subsubsection{UV-Vis spectra}

To further characterize the redox state of PANI of PANI-A-g-EP, UV-Vis was used. Fig. 2 shows the UV-Vis spectra of A- $g$-EP and PANI-A- $g$-EP with various PANI contents. Two peaks at 396 and $580 \mathrm{~nm}$ appear in the spectra of PANI-A-g-EP with 6 and $12 \mathrm{wt} \%$ of PANI, which are assigned to a polaron and a delocalized bipolaron respectively in the partially oxidized state of PANI in the PANI-A-g-EP complex. As PANI content increases, the polaron and delocalized bipolaron increase, resulting the peak intensity of the polaron enhanced. However, on the A$g$-EP and PANI-A- $g$-EP with 3 wt $\%$ of PANI, there is 
neither polaron nor delocalized bipolaron.

\subsection{Surface Resistance}

The surface resistance measurement was used to investigate the anti-electrostatic property. The results are shown in Fig. 6. The surface resistances for the coatings of A- $g$-EP, and PANI(3\%)-A-g-EP, PANI(6\%)-A-g-EP, and PANI(12\%)-A- $g$-EP were $1.6 \times 10^{11}, 1.2 \times 10^{11}, 1.1 \times 10^{7}$, and $2.4 \times 10^{5} \Omega$, respectively. It was generally considered that when the surface resistance was lower than $1.6 \times 10^{9} \Omega$, the coating had an anti-electrostatic property. Luo et al. [26] reported that a surface resistance of a polyurethane silica hybrid coating decreased to $10^{9} \Omega$ by adding $5 \mathrm{wt} \%$ of doped PANI, and a surface resistance in the antielectrostatic range $\left(10^{8} \Omega\right)$ was achieved by incorporating only $10 \mathrm{wt} \%$ of doped PANI (i.e., $1.5 \mathrm{wt} \%$ of PANI). Based on their studies, the present PANI-A- $g$-EP was an intrinsically antistatic coating by bonding no less than 6 $\mathrm{wt} \%$ of PANI on A- $g$-EP.

It is well known that PANI with a high molecule weight has a high electrical conductivity [27]. Though the low molecule weight in PANI-A-g-EP was bad for electrical conductivity, the antistatic property of PANI-A-gEP coating was great, which was expected to come from the delocalized bipolaron in the EB state of PANI in either PANI (6\%)-A-g-EP or PANI (12\%)-A-g-EP. The second possible reason was that PANI doped with a macromolecular acid (A-g-EP-COOH), and the A-g-EP chains served as "conducting bridges", connecting PANI conducting domains and decreasing the percolation value. Similar explanation appeared in the other reports as well $[23,28]$.

\subsection{EIS Measurements and Electroactivity}

Fig. 7 shows Bode impedance plots obtained for the A- $g$-EP coating and the PANI-A-g-EP ones with the PANI solid contents of 3, 6 and $12 \mathrm{wt} \%$ during immersion in 0.1 $\mathrm{M}$ of $\mathrm{H}_{2} \mathrm{SO}_{4}$ solution. As reported in several other papers [29-32], the impedance modulus at $0.1 \mathrm{~Hz}\left(|\mathrm{Z}|_{0.1 \mathrm{~Hz}}\right)$ would be an appropriate parameter for characterizing protective properties of coatings. It was generally considered that a coating with a good anticorrosion performance shall show a low frequency (LF) impedance above $10^{8} \Omega / \mathrm{cm}^{2}$, whereas a poor or failed coating showed a value less than $10^{6} \Omega / \mathrm{cm}^{2}$ [31]. The plots of $|\mathrm{Z}|_{0.1 \mathrm{~Hz}}$ against the immersion time for the coatings are shown in Fig. 7. $|\mathrm{Z}|_{0.1 \mathrm{~Hz}}$ for the A-g-EP coating was $1.2 \times 10^{10} \Omega / \mathrm{cm}^{2}$ after initial immersion, whereas the one for the PANI-A-g-EP coatings with PANI solid content of 3,6 and $12 \mathrm{wt} \%$ was $4.5 \times 10^{8}, 7.5 \times 10^{7}$ and $1.1 \times 10^{7}$ $\Omega / \mathrm{cm}^{2}$, respectively. At the initial immersion, a $|\mathrm{Z}|_{0.1 \mathrm{~Hz}}$ value was related to a charge-transfer resistance $\left(R_{\mathrm{ct}}\right)$ at an electrode material-electrolyte interface. Therefore, the $R_{\mathrm{ct}}$ values of the PANI-A- $g$-EP coatings were much lower than that of the A-g-EP coating, and $R_{\mathrm{ct}}$ deceased as the PANI content increased. This trend was consistent with the effect of the PANI content on the surface resistance shown in Fig. 6 . This result could be due to the delocalized bipolaron in the PANI-A-g-EP, and the density of delocalized bipolaron increased with the increase of PANI content as indicated by the UV results in Fig. 3.

When immersion time increased, the $|\mathrm{Z}|_{0.1 \mathrm{~Hz}}$ of A-g-EP coating decreased to $2.0 \times 10^{9} \Omega / \mathrm{cm}^{2}$ after one day. After 43 days, the $|\mathrm{Z}|_{0.1 \mathrm{~Hz}}$ fell to $1.2 \times 10^{8} / \mathrm{cm}^{2}$, indicating A-g-EP coating had good anticorrosion performance. As compared with ER (E-51) coating, on 40 days, the $|\mathrm{Z}|_{0.1 \mathrm{~Hz}}$ fell below $\times 10^{6} \Omega / \mathrm{cm}^{2}$, indicating failure of protection of the coating [33], A-g-EP coating had better anticorrosion property than pure epoxy coating. In comparison, the $|\mathrm{Z}|_{0.1 \mathrm{~Hz}}$ of PANI (3\%)-A- $g$-EP coating decreased to $\sim 2.0 \times 10^{8} \Omega / \mathrm{cm}^{2}$ during 1-28 days, and decreased to $1.1 \times 10^{6} \Omega / \mathrm{cm}^{2}$ after 43 days, which was higher than $10^{6} \Omega / \mathrm{cm}^{2}$. The $|\mathrm{Z}|_{0.1 \mathrm{~Hz}}$ of PANI (6\%)-A-g-EP decreased to $2.2 \times 10^{6} \Omega / \mathrm{cm}^{2}$ after one day, and maintained this value during the test days, indicating sufficient protective capability of the coating. It was quite different for the PANI (12\%)-A-g-EP that the $|\mathrm{Z}|_{0.1 \mathrm{~Hz}}$ decreased to $3.5 \times 10^{6} \Omega / \mathrm{cm}^{2}$ after one day, decreased gradually to $1.1 \times 10^{5} / \mathrm{cm}^{2}$ during 5-28 days, and increased to $1.0 \times 10^{6} / \mathrm{cm}^{2}$ after 43 day, indicating more efficient passivation of steel and therefore more efficient corrosion protection of the PANI (12\%)-A-g-EP coating.

To further understand the unusual Bode impedance plots obtained for the PANI (12\%)-A-g-EP coating, the electroactivity was investigated. The $\mathrm{CV}$ curves of PANI (3\%)-A-g-EP, PANI(6\%)-A- $g$-EP and PANI(12\%)-A- $g$-EP coatings in $0.10 \mathrm{M}$ of $\mathrm{H}_{2} \mathrm{SO}_{4}$ solution are shown in Fig. 8. There were almost no redox peaks on the $\mathrm{CV}$ curves of PANI (3\%)-A-g-EP, while a pair of redox peaks on the $\mathrm{CV}$ curves of PANI (6\%)-A-g-EP. The oxidation peak was at $0.48 \mathrm{~V}$, and its corresponding reduction peak was weak and at $0.37 \mathrm{~V}$. However, there were two pairs of redox peaks occur on PANI (12\%)-A-g-EP, the first oxidation peak was at $-0.084 \mathrm{~V}$, and its corresponding reduction peak at $-0.15 \mathrm{~V}$. Another oxidation peak was at $0.48 \mathrm{~V}$, and its corresponding reduction peak at $0.40 \mathrm{~V}$, which was much lower than those of PANI $(0.15 \sim 0.2 \mathrm{~V} / \mathrm{SCE}$ and $0.60 \sim$ $0.70 \mathrm{~V} / \mathrm{SCE}$ ) [34]. This deviation could be caused by PANI doped by A-g-EP self-crosslinking macromolecular acid. This low redox potential was favorable for efficient 
passivation of steel.

Scheme 1

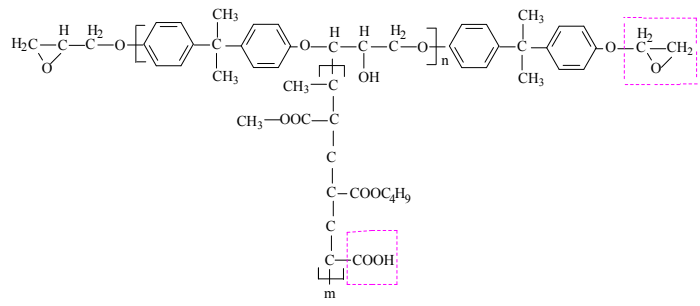

(a)

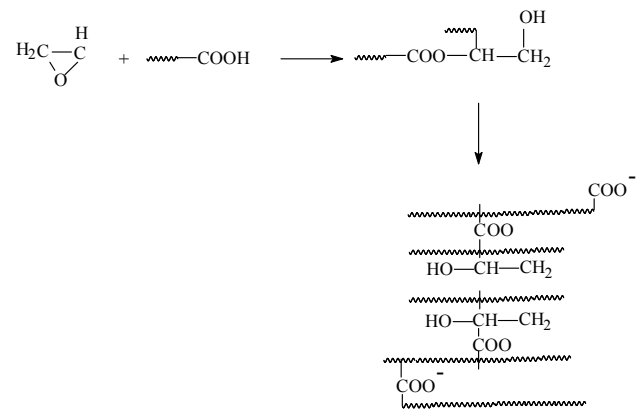

(b)

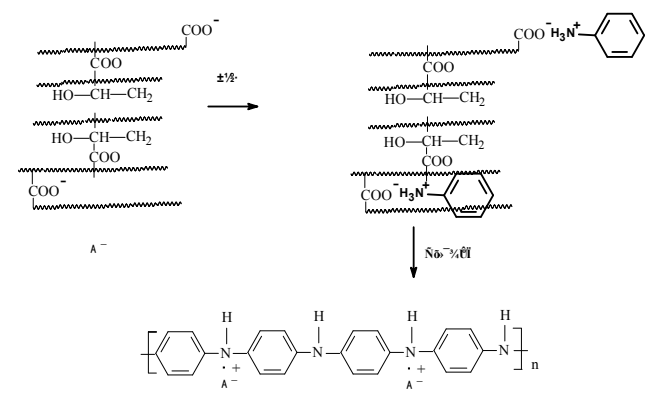

(c)

Scheme 1. (a) The structure of the acrylic ester grafting epoxy; (b) Self-crosslinking reaction of the acrylic ester grafting epoxy; (c) In situ oxypolymerization of self-doped PANI by crosslinked acrylic ester grafting epoxy with carboxylic groups.

Figure 1

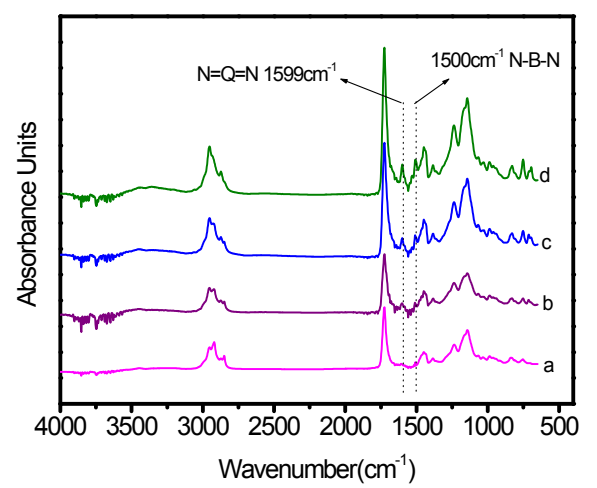

Figure 1.FT-IR spectra of A-g-EP and PANI-A-g-EP complex with different PANI solid content (a) A-g-EP, (b) PANI (3 wt\%)-A-g-EP, (c) PANI (6 wt\%)-A-g-EP, (d)
PANI (12 wt \%)-A-g-EP.

Figure 2

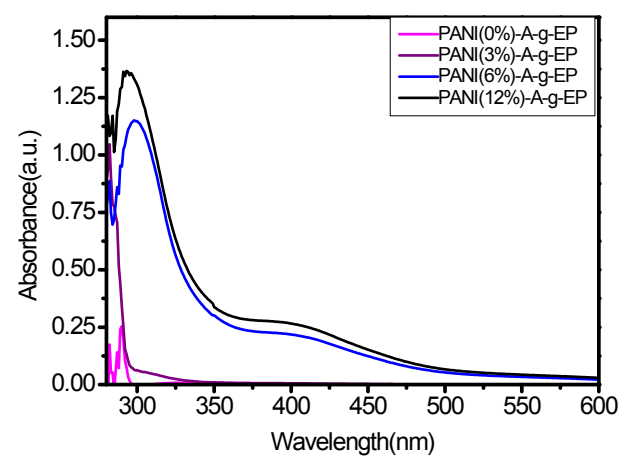

Figure 2. UV-Visible spectra of A-g-EP and PANI-A-g-EP complex

Figure 6

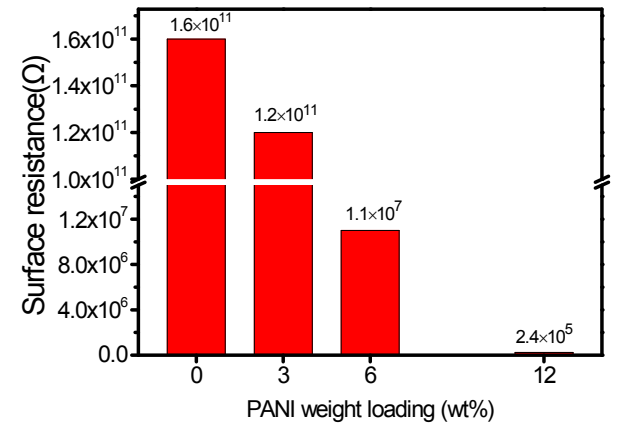

Figure 6. Surface resistance of A-g-EP and PANI-A-g-EP complex with different PANI weight binding.

Figure 7

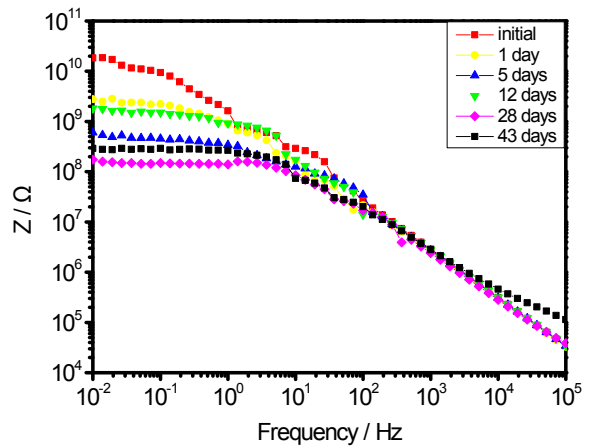

(a) 


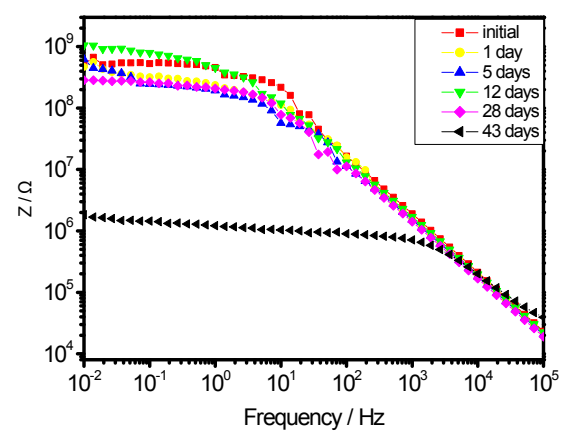

(b)

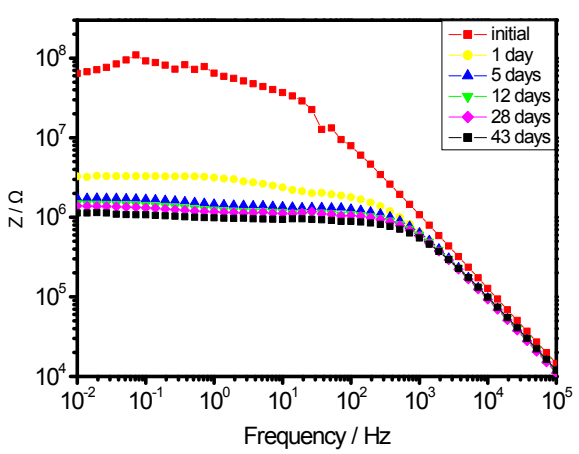

(c)

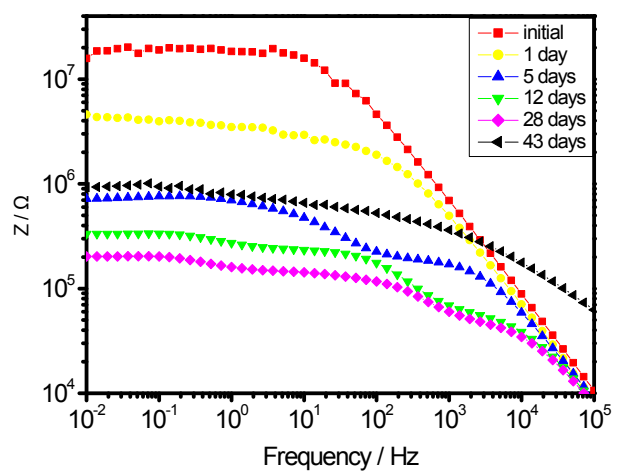

(d)

Figure 7. Bode plots at different immersion time in $0.10 \mathrm{M}$ of $\mathrm{H} 2 \mathrm{SO} 4$ solution for steel coated with (a) A-g-EP, (b) PANI(3\%)-A-g-EP coating, (c) PANI(6\%)- A-g-EP coating and (d) PANI(12\%)- A-g-EP coating

\section{Figure 8}

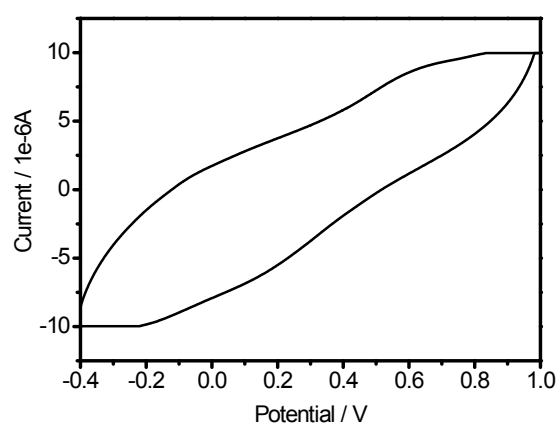

(a)

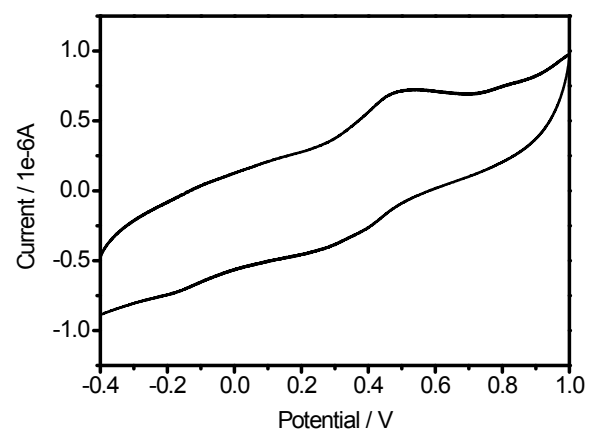

(b)

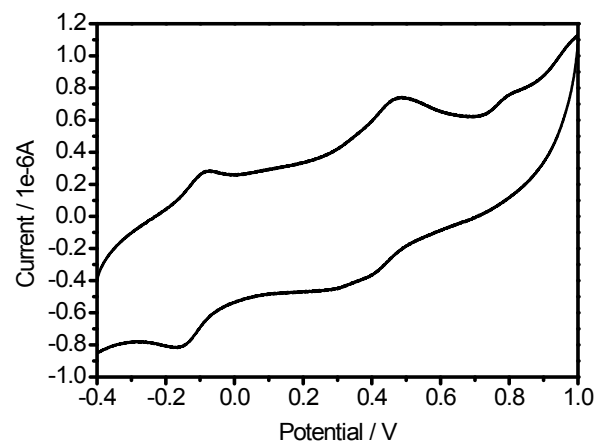

(c)

Figure 8. CV curves of (a) PANI(3\%)-A-g-EP; (b)PANI(6\%)-A-g-EP; (c) PANI(12\%)- A-g-EP coating in $0.10 \mathrm{M} \mathrm{H} 2 \mathrm{SO} 4$ solution.

\section{Conclusions}

PANI-A-g-EP was successfully prepared by in-situ oxidation polymerization of aniline which had been bonded with the carboxyl groups of the A- $g$-EP chain. PANI-A-gEP had a unique self-crosslinking structure. PANI-A-g-EP complex coating showed excellent anticorrosive and antistatic properties. It was the delocalized bipolaron 
existed in PANI-A-g-EP coating, and the delocalized bipolaron density increased with an increase of the PANI content. The PANI-A-g-EP coating with $12 \mathrm{wt} \%$ of the PANI solid content had a good electroactivity, which was favorable for efficient passivation of steel. This PANI-A-gEP complex coating, in which PANI doped with A-g-EP macromolecular acid, provided a novel design of anticorrosive and antistatic complex coating.

\section{Acknowledgment}

This research was financially supported by a grant from the University Science Foundation of Jiangsu province (No. 14KJA430006), Combination of innovative funding (No. SBY2014020171), through the Priority Academic Program Development of Jiangsu Higher Education Institutions and the Propulsion Engineering Program of Industrialization of Research Findings of Jiangsu Higher Education Institutions (JH10-42). The characterizations were conducted at the Testing Center of the Yangzhou University.

\section{References}

(1) P.M. Lesko, P.R. Sperry, Chichester 12, 619, 1997.

(2) S. Muroi. Colloids and Surface A: Physicochemical and Engineering Aspects, 135 (1999) 3.

(3) R. Vera, J. Apablaza, A.M. Carvajal, E. Vera, International Journal of electrochemical science 8 (2013) 11832.

(4) F. Mohtadizadeh, J.M. Zohuriaan-Mehr, Journal of polymer materials 30 (2013) 461.

(5) C.R. Hegedus, F.R. Pepe, J.B. Dickenson, F.H. Walker. J Coat Technol. 74 (2002) 31.

(6) M. Sangermano, W. Carbonaro, G. Malucelli, A. Priola, Macromolecular Material and Engineering 293 (2008) 515.

(7) J. TK Woo, A. Toman, Prog. Org. Coat. 21 (1993) 371.

(8) M. Liu, X.H. Mao, H. Zhu, A. Lin, D.H. Wang, Corrosion Science 75 (2013) 106.

(9) X.M. Wang, J.W. Wang, Q. Li, S.Q. Li, Journal of macromolecular science part B-physics 52 (2013) 751.

(10) J.J. Santana, J.E. Gonzalez, J. Morales, S. Gonzalez, R.M. Souto, International journal of electrochemical science 7 (2012) 6489.

(11) R.M. Torresia, S. de Souza, J. E. Pereira da Silva, S. I. C'ordoba de Torresi, Electrochimica Acta 50 (2005) 2213.

(12) A. Talo, P. Passiniemi, O. Forsén, S. Yläsaaria, Synthetic Metals 85 (1997) 1333.

(13) S. de Souza, Surface \& Coatings Technology 201 ( 2007) 7574.

(14) M.R. Bagherzadeh, F. Mahdavi, M. Ghasemi, H. Shariatpanahi, H.R. Faridib, Progress in Organic
Coatings 68 (2010) 319.

(15) U. Riaz, C. Nwaoha, S.M. Ashraf, Progress in organic coatings 77 (2014) 743 .

(16) N. Birbilis, A.S. Khanna, International journal of electrochemical science 8 (2013) 3132.

(17) C. Dhand, M. Das, M. Datta, B.D. Malhotra, Biosens Bioelectron 26(2011) 2811.

(18) E.S. Matveeva, Synth Met 83 (1996) 89.

(19) M.K. John, R.W. Neloni, H. Justin, W. H. David, Electrochim Acta 51 (2006) 2825.

(20) T. Miroslava, S. Ivana, T. Eva, S. Jaroslav, Polymer Degradation Stability 86 (2004) 179.

(21)C. Zhang, H.M. Zhang, Y.P. Li, J. Li, X.H. Wang, Chinese J. Appl. Chem. 29 (2012) 504.

(22) E. Armelin, R. Pla, F. Liesa, X. Ramis, J. I. Iribarren, C.Alema, Corrosion Science 50 (2008) 721.

(23) Y.F. Ma, S.R. Ali, L. Wang, P.L. Chiu, R. Mendelsohn, H.X. He, J. AM. CHEM. SOC. 128 (2006) 12064.

(24)F.F. Yang, A.P. Zhu, Polymer Bulletin 2014 (in press).

(25) R.F. de Farias, L.M. Nunes, J. Therm. Anal. Calorim, 70 (2002) 559.

(26) J. Luo, X.H. Wang, J. Li, X.J. Zhao, F.S. Wang. Polymer 48 (2007), 4368.

(27) C. Dhand, M. Das, M. Datta, B.D. Malhotra, Biosens Bioelectron 26 (2011) 2811.

(28) Bhavana A. Deore, Insun Yu, Michael S. Freund, J. AM. CHEM. SOC 126 (2004) 52.

(29) A. Amirudin, D. Thierry, Prog. Org. Coat. 26 (1995) 1.

(30) John N. Murray, Prog.Org. Coat. 31 (1997) 375.

(31) A. Shi, S. Koka, J. Ullett, Prog. Org. Coat. 52 (2005) 196.

(32) J.H. Park, G.D. Lee, A. Nishikata, T. Tsuru, Corros. Sci. 44 (2002) 1087.

(33) Y. Chen, X.H. Wang, J. Li, J.L. Lu, F.S. Wang, Corros. Sci. 49 (2007) 3052.

(34) J. Zhang, D. Shan, S.L. Mu, Journal of Polymer Science: Part A: Polymer Chemistry 45 (2007) 5573. 